\title{
Process design in SISO systems with input multiplicity using bifurcation analysis and optimisation
}

\author{
Keming $\mathrm{Ma}^{\mathrm{a} \dagger}$, Héctor Valdés-González ${ }^{\mathrm{b}}$, and I. David L. Bogle ${ }^{\mathrm{a}, *}$ \\ ${ }^{a}$ Centre for Process Systems Engineering, Department of Chemical Engineering, \\ University College London, Torrington Place, London, WC1E 7JE, UK \\ ${ }^{b}$ EUIIIS, University of Tarapacá, Av. 18 de Septiembre 2222, Campus Saucache, Arica, Chile
}

Sept 242009

\footnotetext{
* Corresponding author. Tel.:+44 207679 3803. Fax: +44 2073832348.

E-mail address: d.bogle@ucl.ac.uk (I. David. L. Bogle)

$†$ Current address: MWKellog, Kellogg Tower, Greenford Road, Greenford, Middlesex ,UB6 0JA, UK
} 


\begin{abstract}
This paper presents an approach using continuation and optimisation methods for modifying a process design to avoid control difficulties caused by input multiplicity. The approach assumes an initial design, with a preassigned SISO control structure, has been obtained and is useful where there is an input multiplicity in the operating region. The condition for input multiplicity is obtained by inflating the state space model with a state representing the locus of the point of zero gain. The multiplicity condition is determined using the bifurcation analysis package, AUTO, which allows the study of the influence of operating conditions and parameters on input multiplicity behaviour to obtain an expression for the point of zero gain as a function of the design and disturbance variables. A process modification problem is formulated within an optimisation framework and solved to determine the minimal design parameter changes necessary to avoid input multiplicity given an assumed maximal disturbance. Results are presented for the application of the algorithm to a CSTR system demonstrating that small changes in some design variables can avoid input multiplicity problems in this case, and that the method can determine the changes necessary.
\end{abstract}

Keywords: Process design; Controllability; Nonlinear dynamics; Multiplicity; Process gain singularities; Parametric Optimisation. 


\section{Introduction}

Chemical processes can exhibit complex nonlinear behaviour such as multiplicity. A number of published papers have reported input and output multiplicity found in various chemical processes [1-11].

Input multiplicity occurs when two or more sets of input variables provide the same output conditions. In Jacobsen [8] the implications of input multiplicity for the system dynamics are discussed, and in Koppel [13] and Morari [14] the effect of input multiplicity on the performance of linear controllers are studied with discussion on why input multiplicity imposes more practical control problems than output multiplicity. Sistu and Bequette [12] and Seki and Morari [15] demonstrated control problems caused by input multiplicity behaviour with advanced nonlinear model predictive control. Monnigmann, Marquardt and Hahn [11, 26, 27] presented a method for systematically considering features of nonlinear dynamics in process design and synthesis to alleviate operational problems arising from bifurcations, using bifurcation analysis within an optimisation framework. The optimisation problem seeks to identify a lower bound on the distance of the nominal point of operation to the bifurcation boundary and in Hahn et al. they identify robustness properties of systems under feedback control [27].

Input multiplicity is an inherent characteristic of a process that is determined by the process design. It is now widely recognized that, wherever possible, aspects of process control should be taken into account from the earliest stages of process design. This enables any control difficulties that arise from properties of the process design to be removed by redesigning the process.

This paper presents a method to determine process modifications that will alleviate the control difficulties caused by the input multiplicity in the operating region. This is done by identifying input multiplicity conditions by determining the point of zero gain a change of sign in the gain indicates the point of zero gain - and determining a point where disturbances will not cross the point of zero gain. The proposed method uses tools for bifurcation analysis to locate the point of zero gain, and optimisation to determine the new design. A number of papers have appeared where bifurcation analysis was applied to process design [10, 11, 16-19] and Morari [20] suggests that nonlinear analysis techniques such as bifurcation analysis and singularity theory should be used more routinely in process design to improve controllability. Fortunately, 
numerical bifurcation software packages, such as AUTO [21], make bifurcation analysis of sizeable nonlinear systems practical.

The paper is organised as follows. An input multiplicity detection method using the AUTO tool is presented in section 2. An optimisation problem whose solution determines the process modifications necessary to avoid input multiplicity is formulated and presented in section 3. The analysis and modification of an exothermic reaction as an illustrative example is presented in section 4 , and the main conclusions of the paper are provided in the final section of the paper.

\section{A numerical method for detection of input multiplicity}

Consider a SISO chemical process described by a state-space model of the form:

$$
\begin{aligned}
& \dot{x}=f(x, u) \\
& y=h(x)
\end{aligned}
$$

where $x$ denotes the $n$-vector of state variables, $u$ denotes the manipulated input, and $y$ denotes the controlled output. $f(x)$ and $h(x)$ are smooth vector functions from $\mathbf{R}^{n}$ to $\mathbf{R}^{n}$, whereas $h(x)$ is a smooth scalar function on $\mathbf{R}^{n}$.

Mathematically, a necessary condition for the existence of steady state input multiplicity [13] is:

$$
\mathbf{G}(\mathbf{0})=-\mathbf{C A}^{-1} \mathbf{B}=\mathbf{0}
$$

where $\mathbf{A}, \mathbf{B}$ and $\mathbf{C}$ represent the gradients of function $f(x, u)$ with respect to $x$, $f(x, u)$ with respect to $u$, and $h(x)$ with respect to $x$ at the steady state operating point respectively, and $\mathbf{G ( 0 )}$ is known as the steady state process gain.

Whenever Eq. (2) is true, one must consider the possibility of the existence of input multiplicity behaviour and cannot be confident that the inverse of the system exists as a one-to-one mapping from the values of the output to the values to the input. In this case there is a possibility of a large move of the input for an inverse-based control framework in some circumstances.

Eq. (2) can be used in continuation analysis to determine input multiplicity behaviour of a SISO system when the $u$ varies.

For each given $u$ the system (1) is solved at steady state and then the process gain

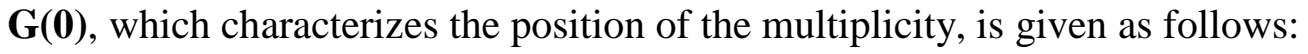




$$
\dot{v}=\mathbf{G}(\mathbf{0}) v
$$

where $v$ is an artificial state and the initial condition is 0 . The value of $\mathbf{G ( 0 )}$ is obtained by solving the system (1) at steady state first for each given $u$.

The eigenvalue of the system (3) is $\mathbf{G ( 0 )}$. A simple singular condition for this particular system at steady-state is:

$$
\mathbf{G}(\mathbf{0})=0
$$

The necessary condition of the existence of input multiplicity is given by a singular point of the gain for this particular dynamic equation. For our purpose, it is sufficient to seek changes in the sign of $\mathbf{G}(\mathbf{0})$ to detect the occurrence of input multiplicity at a variety of steady-states.

If the original dynamic system (1) is augmented with the dynamic equation (3), a new dynamic system is established of the form:

$$
\begin{aligned}
& \dot{x}=f(x, u) \\
& \dot{v}=G(0) v
\end{aligned}
$$

Input multiplicity behaviour of this system can now be detected and located by using bifurcation analysis software. Once located, the input multiplicity dependence on parameter values can be traced out in the space to determine parametric effects. Some preliminary analysis results were presented in [25].

The continuation techniques within the software package AUTO can solve this problem and obtain the locus of the point of zero process gain [21-23], along with bifurcations if they exist.

\section{A method for design modification to avoid input multiplicity}

Once the occurrence of input multiplicity and the locus of the point of zero gain has been determined this can be used within the methodology to determine an improved design.

Consider a given SISO process, which is described by:

$$
\begin{aligned}
& \dot{x}=f(x, u, p, d) \\
& y=h(x)
\end{aligned}
$$


where $x$ is the state vector, $p$ represents the values of the design parameters, $d$ is the maximum disturbance which the design must be able to handle, and $u$ is the manipulated variable.

The input multiplicity condition, as a function of the changes in the manipulated variable, the disturbances, and the design parameters, is obtained using AUTO, and can be expressed as:

$$
u_{\xi}=f_{\xi}(d, p)
$$

This subscript $\xi$ in (7) represents the map of the input multiplicity under variations in the disturbances over the operating range of the input.

Since disturbances can be different for each individual process, a particular value, form or type of maximal system disturbance is assumed and the design modified to account for disturbances up to this magnitude.

The aim of the process modification method is to avoid the control difficulties caused by the input multiplicity behaviour being close to the operating region, in a static sense, i.e., to operate away from the turning point in the process gain (corresponding to a bifurcation point in the system's inverse dynamics) for a disturbance $d$ by adjusting the design parameters. The problem is formulated within an optimisation framework. The objective function is taken to be a measure of how much the design parameters and manipulated variable must move from their original nominal steady-state values. The design parameters are adjusted to keep the output $y$ at its initial point for a set point change to avoid input multiplicity behaviour over the operating range of the manipulated variable.

The scalar objective function $J(u, p, d)$ is defined as a quadratic function that penalises deviations in the manipulated variables and design parameter variables from their nominal steady-state values in a least squares sense:

$$
J(u, p)=\left(p-p^{*}\right)^{T} R\left(p-p^{*}\right)+\left(u-u^{*}\right)^{T} Q\left(u-u^{*}\right)
$$

where $R$ and $Q$ are appropriate weighting factors, and $u^{*}$ and $p^{*}$ are the values of manipulated variable and design parameters at nominal conditions respectively. Thus an 
optimisation problem to determine the design modification to avoid input multiplicity can be formulated as:

$$
\min _{u, p} J=\left(p-p^{*}\right)^{T} R\left(p-p^{*}\right)+\left(u-u^{*}\right)^{T} Q\left(u-u^{*}\right)
$$

subject to

$$
\begin{aligned}
& f(u, x, d, p)=0 \\
& g(u, x, d, p)<0 \\
& y-y_{\text {set }}=0 \\
& \operatorname{sign}\left(u^{*}-f_{\xi}\left(p^{*}, d^{D}\right)\right)\left(u^{*}-f_{\xi}(p, d)\right)<0
\end{aligned}
$$

where $u$ is the manipulated (independent) variable; $x$ is the vector of state variables (dependent); $p$ the vector of independent design variables; $d$ the values of the disturbances; $f$ are the model equations of the process at steady state; $g$ the process constraints; $f_{\xi}$ the input multiplicity condition relation; $d^{D}$ the nominal values of the disturbances; $u^{*}, p^{*}$ the nominal values for the manipulated variable and the design variables; and $y_{\text {set }}$ the set-point value for the controlled variable.

For a given maximal disturbance $d$ the optimisation problem (9) can be solved, resulting in new values of the manipulated variable $\left(u^{\psi}\right)$ and design parameters.

The selected manipulated variable and the design variables are the degrees of freedom for the optimisation. The optimisation takes the nominal solution $u^{*}$ and $p^{*}$ of the process as the base design.

Assuming that the problem (9) has been solved numerically yielding manipulated variables $u^{\psi}$ and design variables $p^{\psi}$ for a disturbance $d$, the modified process at steadystate is expressed as the following:

$$
\begin{aligned}
& f\left(u, x, d, p^{\psi}\right)=0 \\
& y=h(x)
\end{aligned}
$$

which is expected to be able to reject disturbances $d$ successfully in the operating range of the input, improving control performance. The final operating conditions are determined by solving equation (10).

Physically interpreted, the optimisation problem is an attempt to move the operating point away from an input multiplicity condition by using as small as possible a change from the existing design. 
This contrasts with the approach of Marquardt and Monnigmann. They posed a design optimisation problem where the economic objectives are maximised while ensuring that the critical manifold, a surface which separates those steady states which reject disturbances quickly (as defined by the real part of the leading eigenvalue) from those which manage it more slowly, is avoided [11]. This is a procedure based on an open loop stability property. The approach presented here guarantees closed loop stability by the feedback control system design while minimising the economic effect.

\section{An illustrative example: an exothermic CSTR}

This section presents the application of the methodology to an exothermic continuous stirred tank reactor (CSTR) that exhibits input multiplicity. This example demonstrates how the method produces a design which avoids the control difficulties associated with input multiplicity in the operating region.

The method for detection of input multiplicity presented in section 2 is used to determine input multiplicity behaviour of the process and to analyse the effects of the parameters on it. The process modification method developed in section 3 is applied to the process to generate process alternatives.

\subsection{Process description}

Consider a continuous stirred tank reactor with exothermic reaction where an inlet stream to the reactor consisting of pure $\mathrm{A}$ at concentration $c_{A 0}$ and temperature $T_{0}$ enters the reactor, and an exothermic irreversible first-order reaction $A \rightarrow B$ takes place. A cooling jacket kept at a temperature $T_{j}$ is used for the generated heat removal. The effluent stream leaves the reactor at concentrations $c_{A}, c_{B}$ and temperature $T$. The mass and energy balances describing the dynamic behaviour of the process are:

$$
\begin{aligned}
& \dot{C}_{A}=\frac{F}{V}\left(c_{A 0}-c_{A}\right)-k(T) c_{A} \\
& \dot{T}=\frac{F}{V}\left(T_{0}-T\right)+\gamma k(T) c_{A}-\frac{\alpha}{V}\left(T-T_{j}\right)
\end{aligned}
$$

Where $k(T)=k_{0} \exp (-E / R T), \gamma=-\Delta H / \rho C_{p}, \alpha=U A / \rho C_{p}$ 
and the values of the various process parameters and steady-state operating condition are given in Table 1 [24].

The control objective is to control reaction temperature $T$ by manipulating the inlet flow rate $F$. The inlet temperature $T_{0}$ is considered as a disturbance, and the reactor volume $V$ as an adjustable design parameter.

\subsection{Analysis of the process}

The system exhibits input multiplicity and the characteristics were identified by using the method presented in section 2 .

The steady-state solution relationship between the temperature $T$ and the inlet $F$ showing the input multiplicity condition as a result of variation in the inlet temperature $T_{0}$ is given in Fig. 1. The solid line shows the steady-state solution under the nominal conditions, the open square indicates the input multiplicity condition, and the dashed line shows how the inlet temperature $T_{0}$ influences the input multiplicity conditions at steady state during operation. The locus of the input multiplicity condition as a function of the inlet flow rate $F$ and the inlet temperature $T_{0}$ is shown in Fig. 2.

For this process the desired operating point under consideration was chosen to be on the right side of the curve in Fig. 1 to achieve a compromise between maximising conversion and maximising product rate [24]. At the initial steady state operating point, $c_{A}=4.29 \mathrm{kmol} / \mathrm{m}^{3}$ and $T=332 \mathrm{~K}$, the value of the inlet flow rate is $F=0.203 \mathrm{~m}^{3} \mathrm{~h}^{-1}$. As can be seen from Fig. 2, the multiplicity condition could be breached for this selected operating point when there is a decrease in the inlet temperature $T_{0}$ (initially 300K). Fig. 3 gives the input multiplicity condition between the temperature $T$, the inlet feed $F$, and the inlet temperature $T_{0}$ at steady-state. The operating point will move towards or through the curved plane for a decrease in the inlet temperature $T_{0}$, while keeping the reaction temperature $T$ constant. This means that a large decrease in the inlet temperature is likely to cause a control problem as a result of the input multiplicity.

Now we consider the effect of the reactor volume $V$ on the input multiplicity. Figure 4 shows the input multiplicity condition between the inlet flow rate $F$, the inlet temperature $T_{0}$ and the reactor $V$. A decrease in the reactor volume and the inlet temperature will move the operating condition close to the conditions where the control problems associated with the input multiplicity will occur. The results from the analysis 
indicate that a decrease in the inlet temperature $T_{0}$ can cause the input multiplicity condition to be breached during operation.

\subsection{Process design modifications}

The design modification method proposed in section 3 was applied to this process to determine a new design where the input multiplicity condition will not be broached following a disturbance in the inlet temperature. The condition for the existence of input multiplicity between the inlet flow rate $F$, the inlet temperature $T_{0}$ and the reactor volume $V$ is illustrated in Fig. 4. Equation 12 gives the locally linearised relation for the input multiplicity condition between the inlet flow rate, the inlet feed temperature and reactor volume obtained using the data from the bifurcation analysis obtained by AUTO:

$$
F_{I M}=-16.973+0.051 T_{0}+21.195 \mathrm{~V}
$$

For the chosen design variable $V$, the disturbance $T_{0}$, and the manipulated variable $F$, the optimisation problem to be solved to determine the new design is:

$$
\min _{F, V} J=R(F-2.03)^{2}+Q(V-0.1)^{2}
$$

subject to

$$
\begin{aligned}
& \frac{F}{V}\left(c_{A 0}-c_{A}\right)-k_{0} \exp (E / R T) c_{A}=0 \\
& \frac{F}{V}\left(T_{0}-T\right)-\frac{\Delta H}{\rho C_{p}} k_{0} \exp (E / R T) c_{A}-\frac{U A}{\rho C_{p} V}\left(T-T_{j}\right)=0 \\
& T-T_{\text {set }}=0 \\
& F-F_{I M}<0
\end{aligned}
$$

where $R$ and $Q$ are the operating and capital cost coefficients of the changes in the inlet flow rate and reactor volume respectively. In this case $R$ and $Q$ have been given the values of 0.001 and 100 . The values of these weights allow some change to the reactor volume while expecting most change in the inlet flow rate [24].

For an assumed value of the maximal disturbance of the inlet temperature, we obtain a proposed design modification. The design modifications resulting from solving the 
optimisation problem (13) are given in Table 2. In order to have the capacity to reject a change $\Delta T_{0}=-8 \mathrm{~K}$ in the inlet temperature without encountering input multiplicity, the reactor volume $V$ design must be increased from $0.1 \mathrm{~m}^{3}$ to at least $0.111 \mathrm{~m}^{3}$. For a change of $=+2 \mathrm{~K}$ no change in the reactor volume is required which implies that the original process design had the ability to reject this disturbance. The steady-state operating conditions for the proposed process designs for each assumed disturbance are given in Table 3.

\subsection{Closed-loop simulations}

Simulations were undertaken at the initial steady state operating point $\left(c_{A}=4.29 \mathrm{kmol} / \mathrm{m}^{3}\right.$, and $\left.T=332 \mathrm{~K}\right)$ and at the new design conditions. A conventional PI controller was used and its tuning parameters were kept unchanged in all simulation runs for the purpose of comparison. The design changes are small and so any effects on optimal tuning are minimal. All the simulations were done using SIMULINK in MATLAB.

Fig. 5 illustrates the closed-loop response of the reactor temperature $T$ and the profile of the manipulated input $F$ for the original process design, $V=0.1 \mathrm{~m}^{3}$ following a decrease in the inlet temperature $T_{0}$ from $300 \mathrm{~K}$ to $296 \mathrm{~K}$ and from $300 \mathrm{~K}$ to $295 \mathrm{~K}$. The original design $V=0.1 \mathrm{~m}^{3}$ can successfully reject the $-4 K$ step change in the inlet temperature, but cannot for a disturbance of $-5 K$. This is consistent with the analysis above.

In Figure 6 larger decreases in the inlet temperature are imposed for the case of the modified design where $V=0.111 \mathrm{~m}^{3}$. The process can be returned to its initial point in the face of a $-8 K$ change in the inlet temperature.

\section{Conclusions}

A methodology for modifying the design of a process with input multiplicity to avoid the multiplicity, subject to a specified maximum disturbance, has been developed. The proposed method has been applied to an exothermic reactor example. Input 
multiplicity of the process was identified in the parameter space and slight adjustments to the design parameter values improved control performance. The case study demonstrates that the process design modification methodology can generate a new design near the economically optimum design, where the undesired behaviour of the process caused by the input multiplicity can be avoided by adjusting the values of the design parameters.

\section{Acknowledgements}

The authors would like to acknowledge the Engineering and Physical Sciences Research Council of the UK for financial support and Eusebius J. Doedel of Concordia University for help with the use of AUTO. The second author would like also to thank the UTA MAYOR for their support through the project 8710-07. The authors would also like to thank the reviewers for useful suggestions to improve the paper. 


\section{References}

[1] R. Aris, Chemical reactors and some bifurcation phenomena, Ann. NY Acad. Sci. 316 (1979) 314-331.

[2] R. Aris, N. R. Amundson, An analysis of chemical reactor stability and control, Chem. Eng. Sci., 7(3) (1958) 121-131.

[3] V. Balakotaiah, D. Luss, Analysis of the multiplicity patterns of a CSTR, Chem. Eng. Comm. 13 (1981) 111-132.

[4] S. K. Dash, L. B. Koppel, Sudden destabilisation of controlled chemical processes, Chem. Eng. Comm. 84 (1989) 129-157.

[5] W. H. Ray, C. M. Villa, Nonlinear dynamics found in polymerization processes — a review, Chem. Eng. Sci., 55 (2) (2000) 275-290.

[6] A. Uppal, W. H. Ray, A. B. Poore, On the dynamic behaviour of continuous stirred tank reactors, Chemical Engineering Science, 29 (4) (1974) 967-985.

[7] T.E. Güttinger, M. Morari, Predicting multiple steady states in equilibrium reactive distillation. 1. Analysis of nonhybrid systems, Industrial \& Engineering Chemistry Research 38 (4) (1999) 1633-1648

[8] E. W. Jacobsen, Dynamics of systems with steady-state input multiplicity. Proc. AIChE, Annual Meeting, 1994, American Institute of Chemical Engineers.

[9] M. Vaca, A. Jiménez, R. Monroy-Loperenac, On the multiple solutions of the flash equations, Chemical Engineering Science, 61 (12) (2006) 3850-3857.

[10] R. Lemoine-Navaa, A. Flores-Tlacuahuac, E. Saldívar-Guerrac, Non-linear bifurcation analysis of the living nitroxide-mediated radical polymerization of styrene in a CSTR, Chemical Engineering Science, 61(2) (2006) 370-387.

[11] Marquardt W. and Monnigmann M. Constructive nonlinear dynamics in process systems engineering. Comput chem. Engng 29 (2005) 1265-1275.

[12] P.B. Sistu, B. W. Bequette, Model predictive control of processes with input multiplicities, Chemical Engineering Science, 50 (6) (1995) 921-936.

[13] L. B. Koppel, Input multiplicities in nonlinear multivariable control system, AIChE. J., 28 (6) (1982) 935-945.

[14] M. Morari, Design of resilient processing plants III, a general framework for the assessment of dynamic resilience, Chem. Eng. Sci., 38 (11) (1983)1881-1891. 
[15] H Seki, M. Morari, Receding horizon implementation of optimal servo problem: application to a nonlinear process with input multiplicities, Proceedings of the American Control Conference, Philadelphia, PA, USA, (1998) 791-795.

[16] L. P. Russo, B. W. Bequette, Effect of process design on the open-loop behavior of a jacketed exothermic CSTR, Computers \& Chemical Engineering, 20 (4) (1996) 417-426.

[17] L. P. Russo, B. W. Bequette, Operability of chemical reactors: multiplicity behaviour of a jacketed styrene polymerization reactor, Chemical Engineering Science, 53 (1) (1998) 27-45.

[18] W. D. Seider, D. D. Brengel, S. Widagdo, Nonlinear analysis in process design: a review, AIChE Journal, 37 (1) (1991) 1-38.

[19] F. Teymour, W. H. Ray, The dynamic behaviour of continuous solution polymerization reactors-IV. Dynamic stability and bifurcation analysis of an experimental reactor, Chemical Engineering Science, 44 (9) (1989) 1967-1982.

[20] M. Morari, Effect of design on the controllability of chemical plants. In Proc. IFAC Workshop on interactions between process design and process control, London, 1992, International Federation of Automatic Control.

[21] E.J. Doedel, A.R. Champneys, T.F. Fairgrieve, Y.A. Kuznetsov, B. Sandstede, X. Wang, AUTO 97: Continuation and Bifurcation Software for Ordinary Differential Equations (with HomCont), Concordia University, Montreal, Canada, 1998.

[22] E. Doedel, H. B. Keller, J. P. Kernevez, Numerical analysis and control of bifurcation problems. I: Bifurcation in finite dimensions, Int. J. Bifurcation Chaos Appl. Sci. Eng. 1 (3) (1991) 493-520.

[23] E. Doedel, H. B. Keller, J. P. Kernevez, Numerical analysis and control of bifurcation problems. II: Bifurcation in infinite dimensions, Int. J. Bifurcation Chaos Appl. Sci. Eng. 1 (4) (1991) 745-772.

[24] C. Kravaris, P. Daoutidis, R.A. Wright, Output feedback control of no minimum phase nonlinear process, Chem. Eng. Sci., 49 (13) (1994) 2107-2122.

[25] K. Ma and I.D.L. Bogle, Analysing the interaction of open-loop multiplicity and control pairing of nonlinear systems. In Proc. $6^{\text {th }}$ IFAC Symposium on Dynamics and Control of Process Systems, Jejudo Island, Korea, June 2001. 
[26] Monnigmann M. and Marquardt W., Steady state process optimization with guaranteed robust stability and flexibility. AIChEJ 40, (2003) 3110-3126.

[27] Hahn J., Monnigmann M., and Marquardt W., A method for robustness analysis of controlled nonlinear systems. Chem Eng Sci 59 (2004) 4325-4338

Table 1

Parameters and values

\begin{tabular}{lll}
\hline Parameter & Value & Units \\
\hline$k_{0}$ & $7.2 \times 10^{6}$ & $\mathrm{~h}^{-1}$ \\
$R$ & 8.345 & $\mathrm{kJkmol}^{-1} \mathrm{~K}^{-1}$ \\
$-\Delta H$ & $7.0 \times 10^{4}$ & $\mathrm{kJkmol}^{-1}$ \\
$U A$ & 1680 & $\mathrm{kJh}^{-1} \mathrm{~K}^{-1}$ \\
$V$ & 0.1 & $\mathrm{~m}^{3}$ \\
$c_{A 0}$ & 10 & $\mathrm{kmolm}^{-3}$ \\
$E$ & $4.1 \times 10^{4}$ & $\mathrm{kJkmol}^{-1}$ \\
$C_{p}$ & 4.2 & $\mathrm{kJkg}^{-1} \mathrm{~K}^{-1} \mathrm{~h}^{-1}$ \\
$\rho$ & 1000 & $\mathrm{kgm}^{-3}$ \\
$T_{j}$ & 300 & $\mathrm{~K}^{2}$ \\
$T_{0}$ & 300 & $\mathrm{~K}$ \\
\hline
\end{tabular}

Table 2

Process design modification results for the exothermic reaction

\begin{tabular}{lllllll}
\hline$\Delta T_{0}$ & -10 & -8 & -6 & -4 & 0 & +2 \\
$V$ & 0.116 & 0.111 & 0.106 & 0.101 & 0.100 & 0.100 \\
\hline
\end{tabular}

Table 3

Parameters and values

\begin{tabular}{lllll}
\hline Parameter & Base & Alt. 1 & Alt.2 & Alt.3 \\
\hline$\Delta T_{0}(K)$ & -4 & -6 & -8 & -10 \\
$V\left(m^{3}\right)$ & 0.100 & 0.106 & 0.111 & 0.116 \\
$T_{s}(K)$ & 332 & 332 & 332 & 332 \\
$F_{s}\left(m^{3} h^{-1}\right)$ & 0.203 & 0.190 & 0.177 & 0.169 \\
$c_{A, s}\left(\mathrm{kmolm}^{-3}\right)$ & 4.30 & 4.05 & 3.74 & 3.52 \\
\hline
\end{tabular}




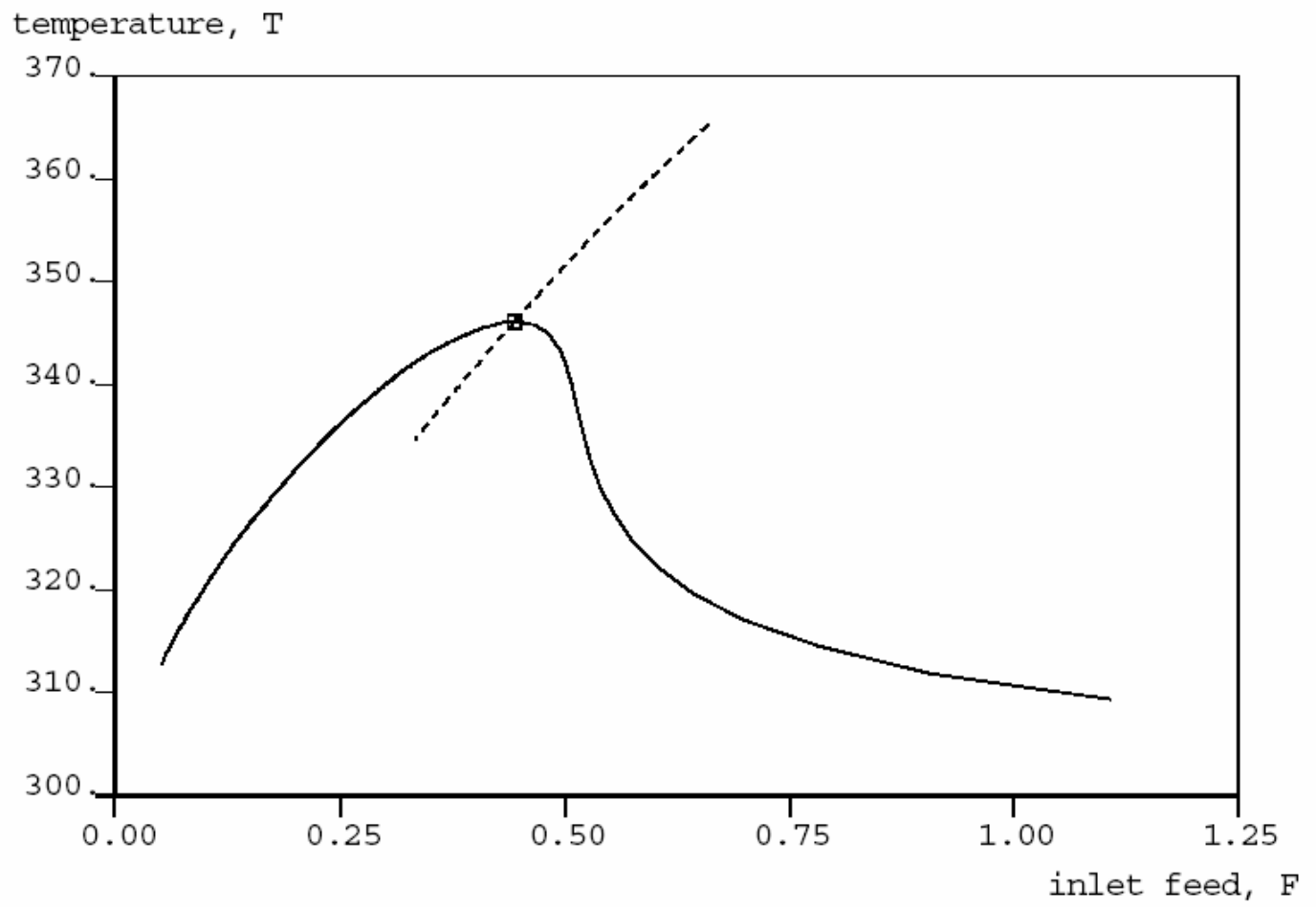

Fig. 1. Steady-state solutions showing input multiplicity condition with variations of inlet temperature. Square: input multiplicity condition; dashed line: input multiplicity condition with variation of inlet temperature. 


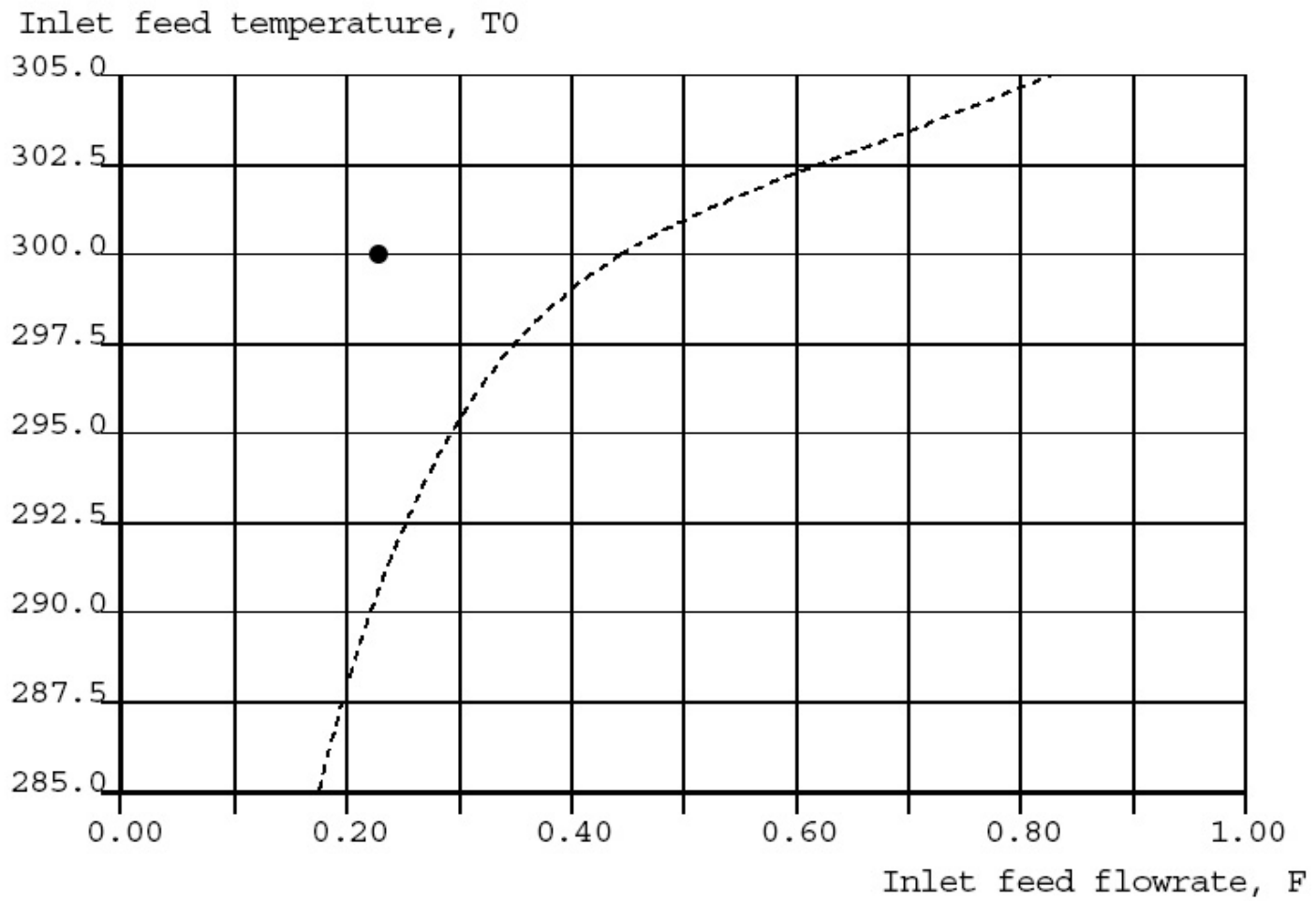

Fig. 2. Locus of inlet flow rate versus inlet temperature at input multiplicity condition.

The point $\bullet$ indicates locus of the initial designed operating point.

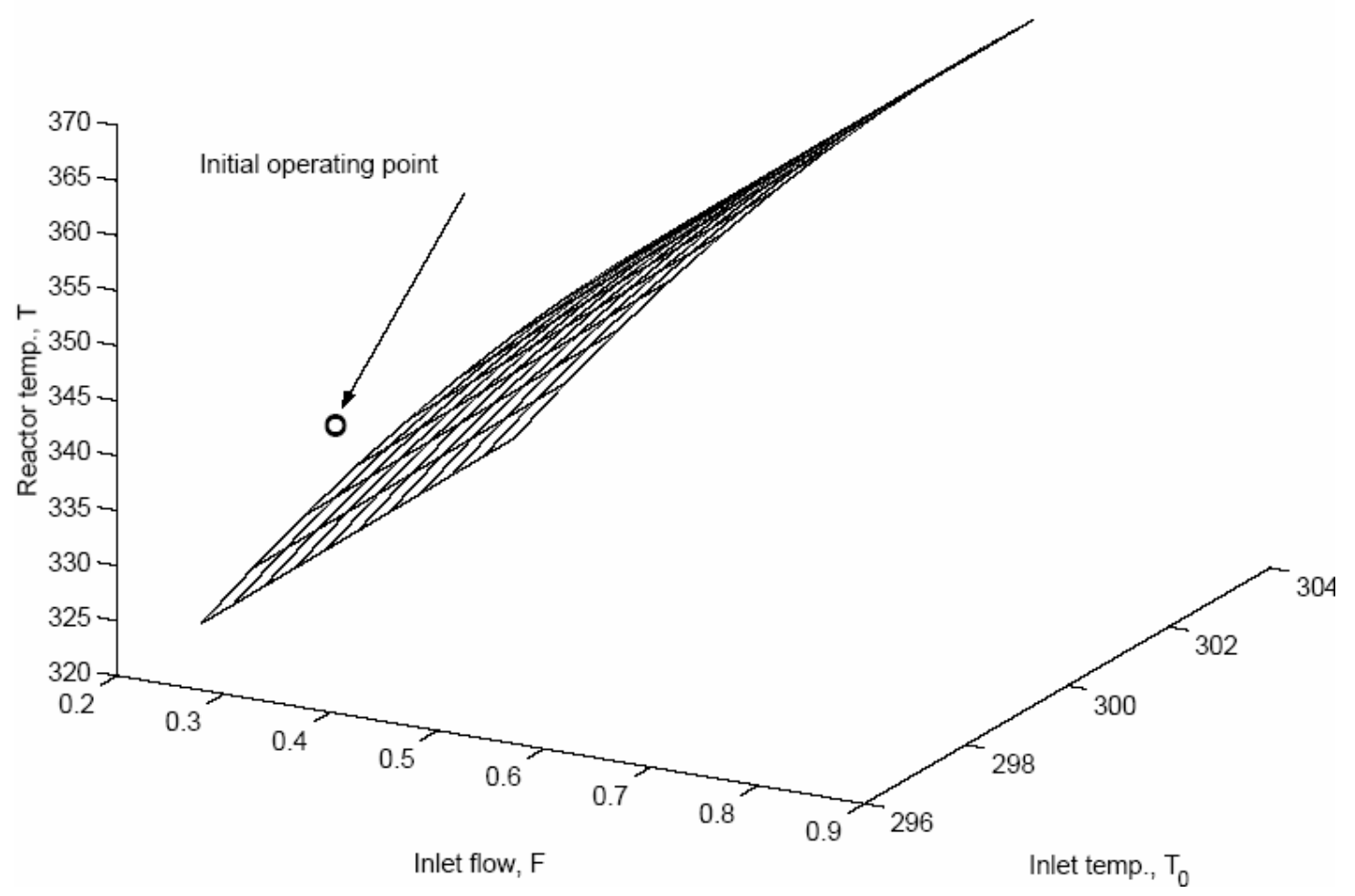


Fig. 3. Locus of input multiplicity condition between the rector temperature, inlet flow rate and inlet temperature

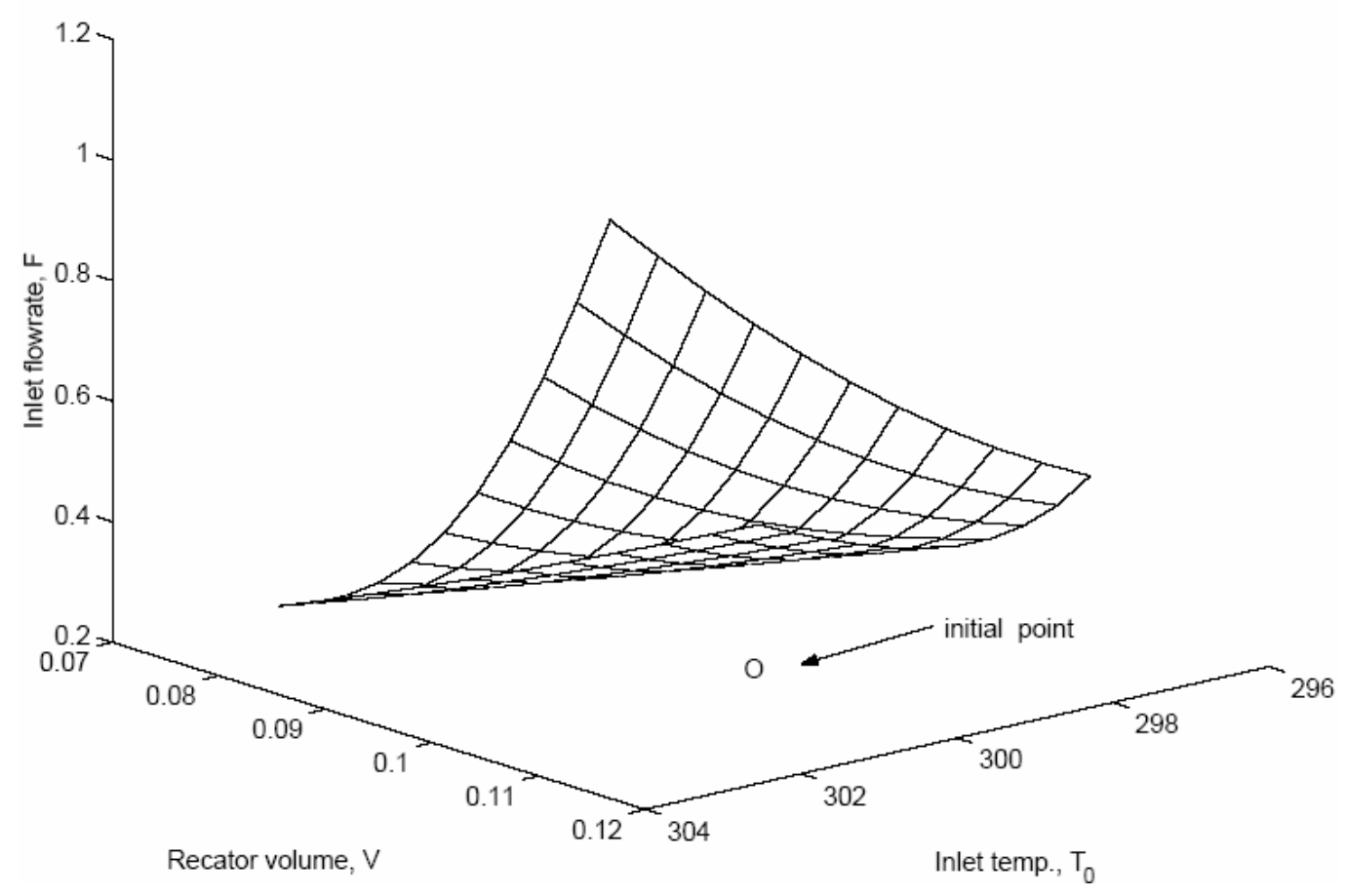

Fig. 4. Locus of input multiplicity condition between the inlet flow rate, inlet temperature and reactor volume 

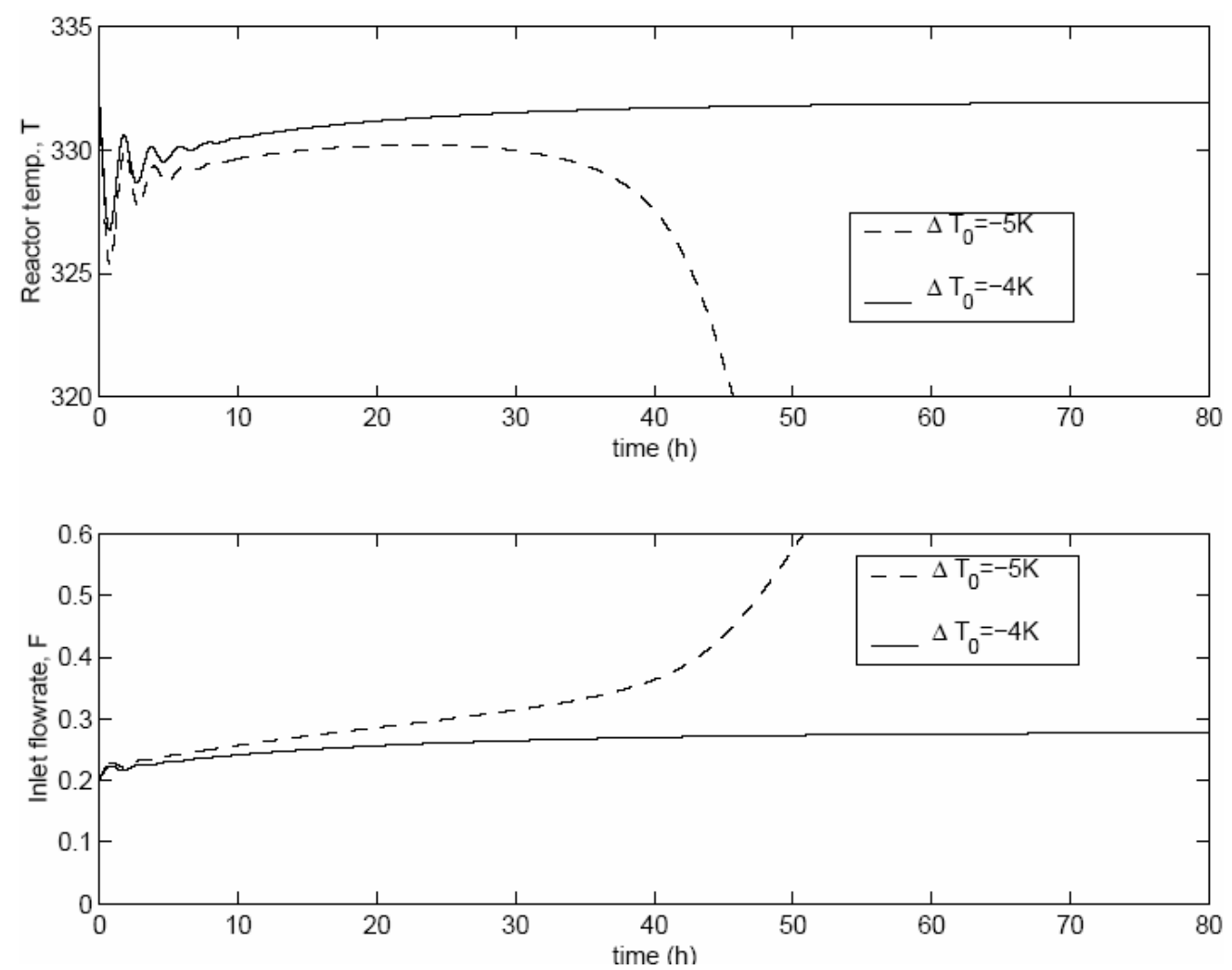

Fig. 5. Profiles of input and output for the initial design, $V=0.1 \mathrm{~m}^{3}$, for disturbance rejection of inlet temperature change of $-4 K$ and $-5 K$
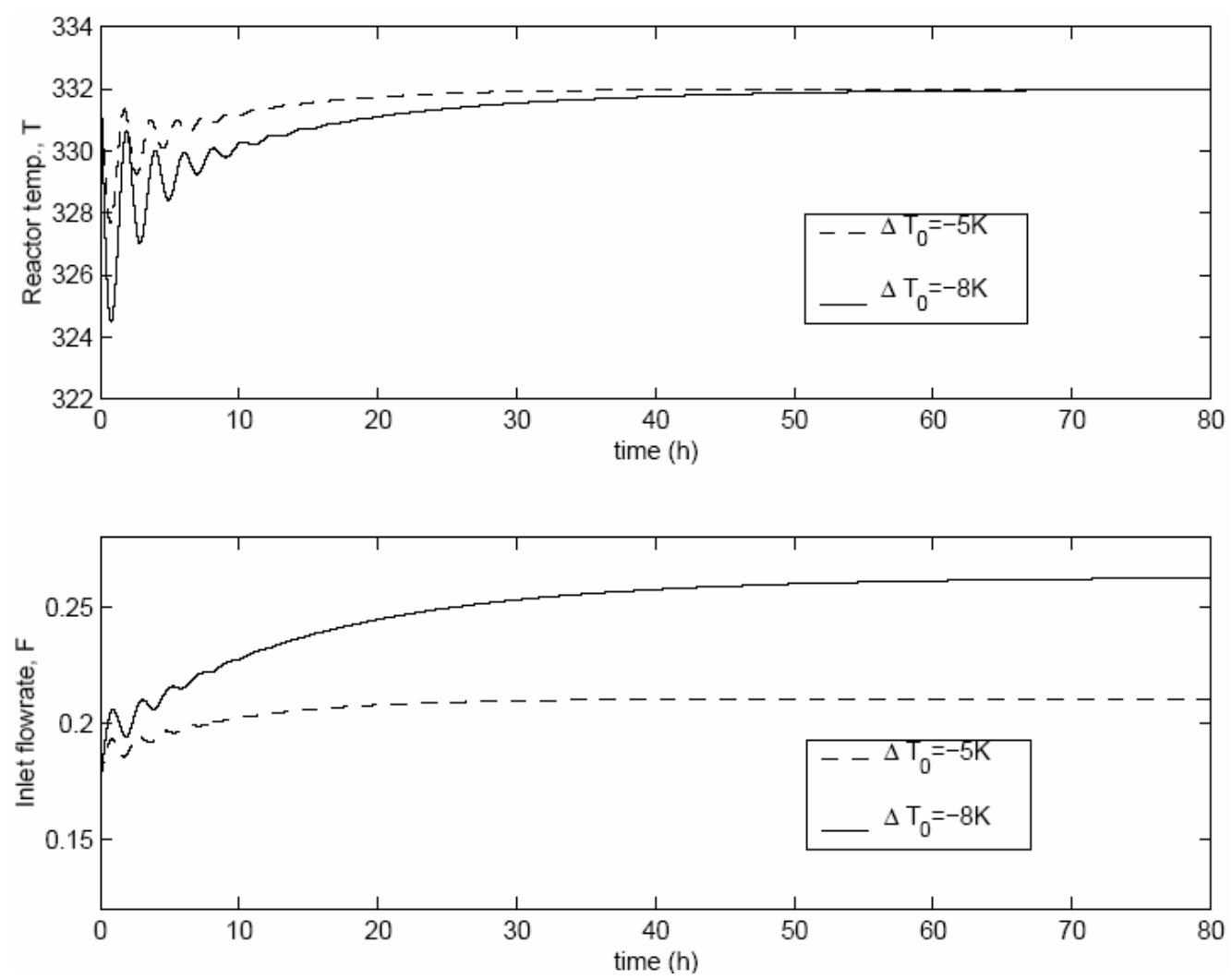

Fig. 6. Profiles of input and output for the modified design, $V=0.111 \mathrm{~m}^{3}$, for rejection of inlet temperature changes of $-5 K$ and $-8 K$ 